\title{
Cava of the ventricular system
}

\section{R. Shane Tubbs}

Received: 5 August 2011 /Accepted: 11 August 2011 /Published online: 23 August 2011

(C) Springer-Verlag 2011

\section{Dear Editor,}

We thank this reader for the comments regarding our paper. The reader rightfully points out legend errors. The terminology of such cava varies widely in the literature. We agree that the history behind these fluid-filled structures is interesting.

Sincerely,

R. Shane Tubbs

Birmingham, AL, USA 